\title{
Stereoselective Synthesis of Enantiopure 4,5-Dihydroxy-2-Alkene Esters from Simple Allylic Alcohols
}

\author{
Marcel P.M. van Aar, Iambertus Thijs and Binne Zwanenburg* \\ Department of Organic Chemistry, NSR Center for Molecular Structure, Design and Synthesis, \\ University of Nijmegen, Toernooiveld, 6525 ED Nijmegen, The Netherlands
}

\begin{abstract}
A general stereoselective synthesis of 4,5-dihydroxy-2-alkene esters is developed using the photo-induced rearrangement of $\alpha, \beta$-epoxy diazomethyt ketones. Starting with readily available enantiopure allylic alcohols that contain a chiral center at $C_{\Delta}$. i.e. a protected secondary alcohol function, a neighboring stereogenic center is introduced by irradiation of the mentioned diazo ketones. The configuration of this newly introduced center is determined by the chiral inductor used in the Sharpless epoxidation of the allylic alcohol and therefore can be selected at will.
\end{abstract}

The photo-induced rearrangement of $\alpha, \beta$-cpoxy diazomethyl ketones constitutes an attractive synthetic method for the preparation of $\gamma$-hydroxy- $\alpha, \beta$-unsaturated esters $1^{1.2}$. The starting materials for epoxy diazo ketones are allylic alcohols which by Sharpless epoxidation ${ }^{3}$ are converted into 2,3-epoxy alcohols. Subsequent oxidation of these alcohols gives oxirane-2-carboxylic acids of high enantiopurity. Transformation into the corresponding epoxy diazo ketones can readily be accomplished using a series of standard operations ${ }^{4}$. The photochemical conversion into $\gamma$-hydroxy-alkene esters is carried out in alcoholic solvents. It is of importance to note that the chiral integrity of the stereogenic center at $\mathrm{C}_{3}$ of the starting material is retained in the product ${ }^{2}$. The sequence of events is depicted in scheme 1.

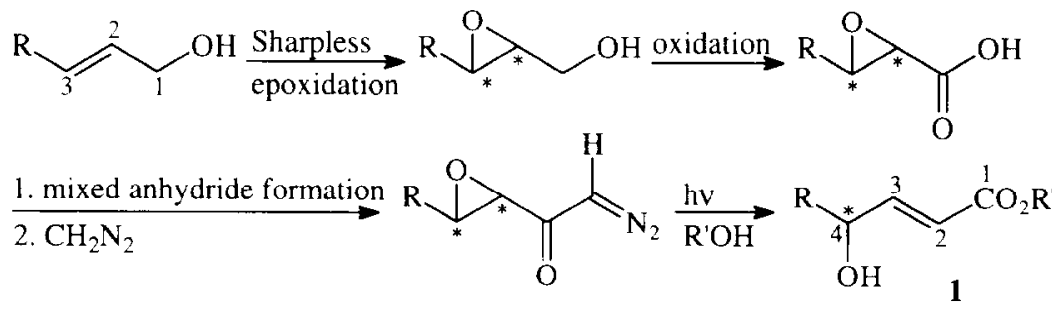

Scheme I

This methodology has been applied in the synthesis of several naturally occurring macrocyclic lactones, such as $(+)$-aspicilin', pyrenophorol ${ }^{6}$, colletallol ${ }^{7}$, patulolid $\mathrm{C}^{8,9}$, isopatulolid $\mathrm{C}$ and analogues ${ }^{9}$ and the macrocyclic subunit of cytochalasin $B^{10}$, all containing the 4-hydroxy-2-alkene ester moiety. Hydrogenation of 
the $\gamma$-hydroxy-alkene esters 1 gives $\gamma$-hydroxy carboxylic esters, which readily give ring closure to yield enantiopure $\gamma$-lactones ${ }^{4}$. This spin-off of the preparation of $\gamma$-hydroxy-alkene esters has successfully been applied in the synthesis of the naturally occurring $\gamma$-lactone rubrenolide ${ }^{11}$. An interesting question arises whether it will be possible to introduce a new stereogenic center, adjacent to an already existing one, without affecting the chiral integrity of this neighboring center, by the method shown in scheme 1 . The synthetic outline for this planned series of operations is depicted in scheme 2. To execute this plan it is convenient to start with suitably protected allylic alcohols, which already contain a stereogenic center, preferably a secondary hydroxyl function. By a proper choice of the chiral inductor in the Sharpless epoxidation the configuration of the newly introduced chiral center can he selected at will.

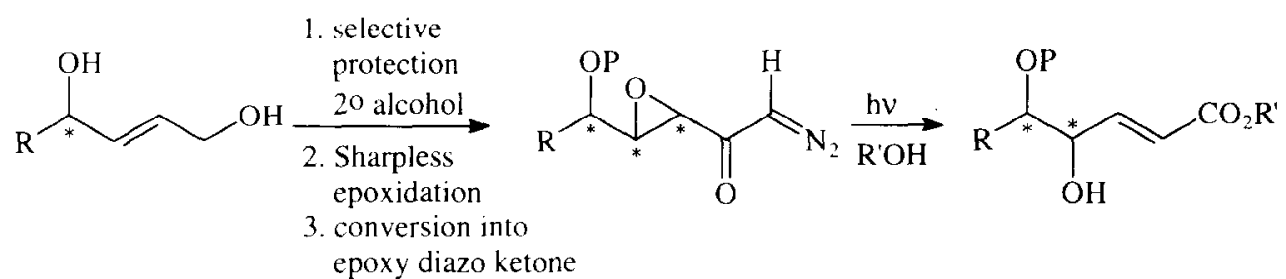

Scheme 2

D-Mannitol and L-malic acid were chosen as starting materials for the preparation of allylic alcohols containing a stereogenic center. (R)-isopropylidene glyceraldehyde 2 can readily be obtained from D-mannitol by a glycol cleavage procedure that is well documented ${ }^{12.13}$. Allylic alcohol 3 was prepared from this aldehyde in a iwo-step process, viz. a Wittig-Horner type chain elongation with triethyl phosphonoacetate ${ }^{14}$ (E/Z-ratio 25:1), followed by a reduction with DIBAL-H (scheme 3). By following the sequence of reactions shown in

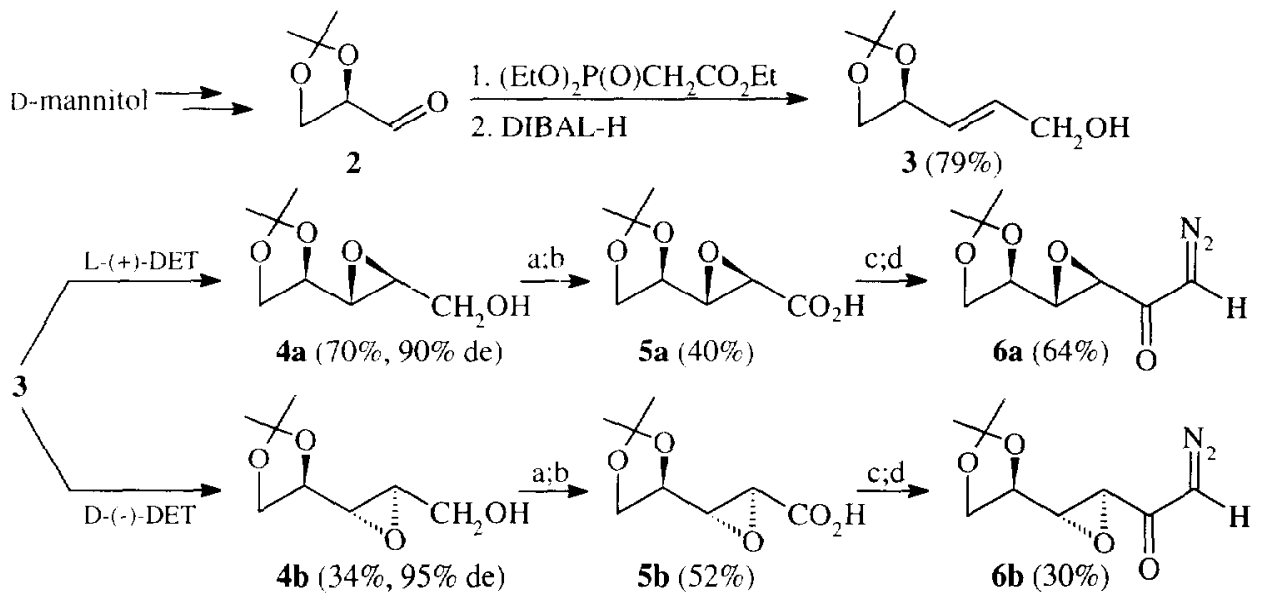

a. $\mathrm{CrO}_{3} /$ pyridine/ $\mathrm{CH}_{2} \mathrm{Cl}_{2}$; b. PDC/DMF; c. $\mathrm{ClCO}_{2} \mathrm{iBu} / \mathrm{Et}_{3} \mathrm{~N}$; d. $\mathrm{CH}_{2} \mathrm{~N}_{2}$ 
scheme 2, epoxy diazomethyl ketones $\mathbf{6 a}$ and $\mathbf{6 b}$ were synthesized. Asymmetric epoxidation of allylic alcohol 3 to the epoxy alcohols $\mathbf{4 a}$ and $\mathbf{4 b}$ was realized by using either $\mathrm{L}-(+)$-DET or D-(-)-DET as chiral inductor ${ }^{15,16,17}$. Both epoxidations showed a high diastereomeric excess. as was determined by capillary gas chromatography (cf. ref. ${ }^{18}$ ). Catalytic one-step oxidation of $\mathbf{4}$ to 5 , employing $\mathrm{RuO}_{4}$ in aqueous tetrachloromethane/acetonitrile ${ }^{19}$, gave unsatisfactory results as undefined products in low yields were obtained. Therefore, the oxidation to the carboxylic acids 5 had to be performed in a two-step procedure using non-aqueous conditions because of the high water-solubility of compounds $\mathbf{4}$ and $\mathbf{5}^{17}$. It was found that Collins's reagent ${ }^{20}$ is suitable for the conversion to the aldehyde and that pyridinium dichromate ${ }^{21}$ gives satisfactory results in the second oxidation to the carboxylic acids 5. For the preparation of the diazo ketones 6 the standard procedures ${ }^{4}$ could be used successfully.

Irradiation of compounds 6 in methanol leads to alkene esters 7, which both have two well-defined stereogenic centers (scheme 4). In the ${ }^{1} \mathrm{H}$-NMR spectrum of the products $7 \mathbf{a}$ and $\mathbf{7 b}$ a difference was observed

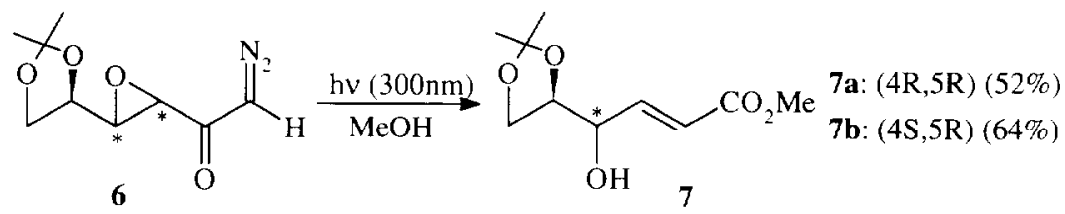

Scheme 4

for the chemical shift of the $C_{4}$-protons. In 7a this signal is part of the multiplet at 3.83-4.30 ppm, while in $7 \mathbf{b}$ this signal appears at $4.48 \mathrm{ppm}$. Compound $7 \mathbf{b}$ was synthesized previously by Regeling and Chittenden ${ }^{22}$ from D-glucono-1,5-lactone. The spectral features are in full accordance. The spectra of the products 7 showed no signals of the other epimer, which means that the stereochemical configuration at $\mathrm{C}_{4}$ is retained during the synthetic sequence starting with 4 . It should be noted that by starting from enantiomeric (S)-isopropylidene glyceraldehyde ${ }^{23}$ the (4R,5S) and (4S,5S) diastereomers of 7 are also accessible.

As a sccond chiral substrate for this study, cyclic acetal 8 was selected (sclieme 5). This compound can conveniently be obtained from L-malic acid by borane-dimethyl sulfide reduction according to Hanessian et $a l^{24}$, followed by a trans-acetalization with benzaldehyde dimethyl acetal. In this manner the six-membered ring acetal $\mathbf{8}$ is formed regioselectively (no five-membered ring product was present) displaying the (S)configuration at the phenyl substituted carbon atom ${ }^{25}$. Swern oxidation ${ }^{26}$ of 8 leads to an aldehyde suitable for chain elongation by a Wittig-Horner type reaction (E/Z-ratio 20:1). Reduction of the alkene ester obtained with DIBAL-H produces allylic alcohol 9. This secondary hydroxyl protected compound is used as substrate for the synthetic plan shown in scheme 2. Sharpless epoxidation with either L-(+)-DET or D-(-)-DET as chiral inductors gave epoxy alcohols 10 (both reactions proceeded with high diastereomeric excess, as was determined by capillary gas chromatography), which in turn were converted into carboxylic acids 11, using again a two-step procedure, viz. Swern oxidation ${ }^{26 \mathrm{~b}}$ to the aldehyde and subsequent oxidation with sodium chlorite ${ }^{27}$ to the carboxylic acid. An attempted one-step oxidation of $\mathbf{1 0}$ using ruthenium tetroxide ${ }^{19}$ did not give satisfactory results, due to oxidation of the phenyl ring. The two-step procedure mentioned above is different from that used for the conversion of $\mathbf{4}$ into $\mathbf{5}$, due to the low solubility of $\mathbf{1 0}$ and $\mathbf{1 1}$ in water. During the work-up of $\mathbf{1 1}$, acidification was very critical, because of the acid-sensitivity of the acetal function in the dioxane ring. This 

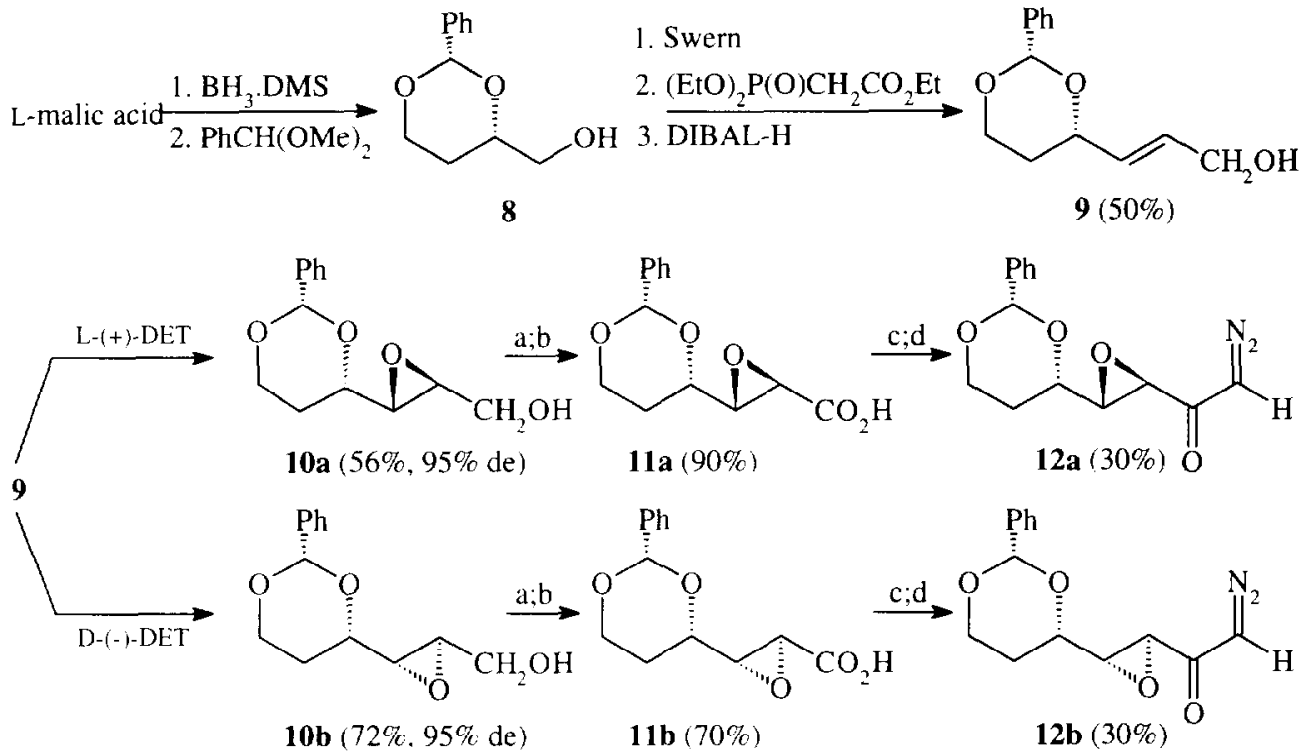

a. $(\mathrm{ClCO})_{2} / \mathrm{DMSO} ;$ b. $\mathrm{NaClO}_{2} / \mathrm{NaH}_{2} \mathrm{PO}_{4} ;$ c. ClCO $2 \mathrm{Bu} / \mathrm{Ft} \mathrm{C}_{3} \mathrm{~N}$; d. $\mathrm{CH}_{2} \mathrm{~N}_{2}$

Scheme 5

sensitivity towards acid also played a role during chromatography over silica gel. Diazo ketones $\mathbf{1 2}$, which were synthesized from 11 without any difficulty, could be isolated but a considerable loss had to be accepted due to decomposition and rather large amounts of benzaldehyde were isolated as well.

Irradiation of 12 in methanol solution gave alkene esters 13 with defined stereogenic centers at $\mathrm{C}_{4}$ and $\mathrm{C}_{5}$ (scheme 6). In the ${ }^{1} \mathrm{H}$-NMR spectra of $\mathbf{1 3 a}$ and $\mathbf{1 3 b}$ a considerable difference for the signals of the $\mathrm{C}_{4}$ protons was observed. In 13a this proton gives a multiplet at $4.41-4.53 \mathrm{ppm}$, whereas in 13b it is part of the multiplet at 4.18-4.38 ppm. In comparison with $7 \mathbf{a}$ and $\mathbf{7 b}$, the stereogenic center at $C_{5}$ of compounds $\mathbf{1 3 a}$ and $\mathbf{1 3 b}$ has an opposite absolute configuration. $\Lambda$ s a consequence the NMR spectrum for the $C_{4}$ and $C_{5}$ protons of $7 \mathbf{a}$<smiles>N#CC(=O)C1O[C@H]1C1CCOC(c2ccccc2)O1</smiles>

12<smiles>COC(C)=CC=C(O)[C@@H]1CC[C@H](CO)OC1c1ccccc1</smiles>

13 13a: $(4 \mathrm{R}, 5 \mathrm{~S})(50 \%)$

13b: $(4 S, 5 S)(75 \%)$

Scheme 6 
resembles that of $\mathbf{1 3 b}$ and the same holds for $7 \mathbf{b}$ and $13 \mathbf{a}$. As with products 7 , the NMR spectra of compounds 13 show no signals of the other epimer, indicating that the configuration at $\mathrm{C}_{4}$ is retained during the synthetic operations starting from 10 . It should be noted that when enantiomeric D-malic acid would be used as starting material the (4R,5R) and (4S,5R) diastereomers of 13 are accessible too.

As mentioned, in both sets of experiments the diastereomeric excess in the epoxidation reaction using either L-(+)-DET or D-(-)-DET is high. Using the same starting allylic alcohol and epoxidation method, Sharpless et al. ${ }^{18}$ reported a diastereomeric excess of 95.6 and $98.9 \%$ for the epoxy alcohols $\mathbf{4 a}$ and $\mathbf{4 b}$, respectively. However, no experimental details were reported. From synthetic point of view our results indicate that in the latter (D-(-)-DET) case the chiral center at $\mathrm{C}_{4}$ of allyl alcohol 3 cooperates with the chiral auxiliary in the epoxidation reaction, whereas in the former $(\mathrm{L}-(+)-\mathrm{DET})$ case there is no significant influence of the chiral center at $\mathrm{C}_{4}$ on the chiral induction.

In conclusion, the two investigated allylic alcohols derived from D-glyceraldehyde and L-malic acid, both containing a protected secondary alcohol function as stereogenic center, can be stereoselectively converted into enantiopure 4,5-dihydroxy-2-alkene esters using the photo-induced rearrangement of $\alpha, \beta$-epoxy diazomethyl ketones. This synthetic strategy may be useful in the constuction of molecules, for instance natural products, with hydroxy groups attached to two different stereogenic centers.

\section{EXPERIMENTAL SECTION}

General remarks:

${ }^{1} \mathrm{H}-\mathrm{NMR}$ spectra were recorded on a Varian EM $390(90 \mathrm{MHz}, \mathrm{CW})$, a Bruker AC-100 (100 MHz, FT) or a Bruker AM-400 (400 MHz., FT) spectrometer with TMS as internal standard. ${ }^{13} \mathrm{C}$-NMR spectra were recorded on a Bruker AM-400 (100 MHz, FT) spectrometer with $\mathrm{CHCl}_{3}$ as internal standard. IR spectra were run on a Perkin-Elmer 298 spectrophotometer. Elemental analyses were standard carried out in triplicate on a Carlo Erba Instruments CHNSO EA 1108 element analyzer. For mass spectroscopy a double focusing VG 7070E was used. For the chemical ionization (CI) technique, methane was used as reacting gas. Melting points were measured on a Reichert Thermopan microscope and are uncorrected. Optical rotations were determined on a Perkin-Elmer 241 polarimeter. GC was performed on a Hewlett-Packard 5890 or a Hewlett-Packard 5890 Series II instrument, equipped with a capillary HP cross-linked methyl silicone (25 m x $0.31 \mathrm{~mm}$ ) column, connected to a HP 5890 calculating integrator. For chromatography the flash technique was used with silica gel $60 \mathrm{H}$ (Merck) as stationary phase and a pressure of about 1.5 bar. Chromatography over florisil was performed under normal pressure. Chemical compounds were named using the Autonom ${ }^{3}$ program, version 1.0. All solvents used were dried and distilled according to standard procedures. When diazomethane was used, proper safety precautions were taken.

E-3-l(4R)-2,2-dimethyl-1,3-dioxolan-4-vl]-prop-2-en-l-ol (3):

To a stirred suspension of oven-dried $\mathrm{LiCl}(6.9 \mathrm{~g}, 175 \mathrm{mmol})$ in dry acetonitrile $(140 \mathrm{ml})$ were successively added triethyl phosphonoacetate $(37.0 \mathrm{~g}, 175 \mathrm{mmol})$. N.N-diisopropylethylamine (DIPFA, $25.6 \mathrm{ml}, 146 \mathrm{mmol}$ ) and aldehyde $2(19.0 \mathrm{~g}, 146 \mathrm{mmol})^{12.13}$ under nitrogen and at room temperature. During the addition of 2 the mixture became cloudy and warm. The reaction was followed by TLC and after $1 \mathrm{~h}$ the aldehyde had disappeared. Water was added until the solution became clear and acetonitrile was evaporated. The residue, a 
cloudy oil, was taken up in water and extracted with ether $(4 \mathrm{x})$. After drying $\left(\mathrm{MgSO}_{4}\right)$ the combined organic layers were concentrated to give $33.98 \mathrm{~g}$ of crude product. Of this mixture $12.0 \mathrm{~g}$ was chromatographed (hexane/ethyl acetate $4: 1$ ) yielding pure trans-ester as an oil $(8.54 \mathrm{~g}, 83 \%)$. ' $\mathrm{H}-\mathrm{NMR}\left(\mathrm{CDCl}_{3}, 100 \mathrm{MHz}\right): \delta 1.22$

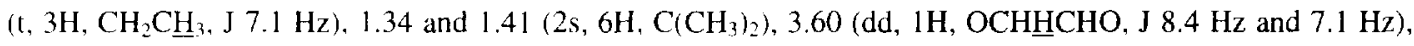
4.11 (dd, 1H, OCLHHCHO, J $8.4 \mathrm{~Hz}$ and $\mathrm{J} 6.5 \mathrm{~Hz}), 4.14\left(\mathrm{q}, 2 \mathrm{H}, \mathrm{C}_{2} \mathrm{CH}_{3}, \mathrm{~J} 7.1 \mathrm{~Hz}\right), 4.60\left(\mathrm{~m}, 1 \mathrm{H}, \mathrm{OCH}_{2} \mathrm{CHO}^{\mathrm{H}}\right.$ ), $6.02(\mathrm{dd}, 1 \mathrm{H}, \mathrm{CH}=\mathrm{CHCO} . \mathrm{J} 15.5 \mathrm{~Hz}$ and $1.3 \mathrm{~Hz})$ and $6.82(\mathrm{dd}, 1 \mathrm{H}, \mathrm{CH}=\mathrm{CHCO}, \mathrm{J} 15.5 \mathrm{~Hz}$ and $\mathrm{J} 5.5 \mathrm{~Hz}) \mathrm{ppm}$. IR $\left(\mathrm{CCl}_{4}\right): v 2980,2940,2870,1720,1660,1380.1370,1300,1250 \mathrm{~cm}^{-1}$. Also pure cis-ester was isolated as an oil $(0.62 \mathrm{~g}, 6 \%)$. The trans-ester $(8.0 \mathrm{~g}, 40 \mathrm{mmol})$ was dissolved in dried ether $(125 \mathrm{ml})$ under nitrogen at $0^{\circ} \mathrm{C}$. DIBAL $\mathrm{H}$ was added using a syringe $(80 \mathrm{ml}$ of a $1.0 \mathrm{M}$ solution in hexane). After $15 \mathrm{~min}$ the ester had disappeared (TLC) and $\mathrm{Na}_{2} \mathrm{SO}_{4} \cdot 10 \mathrm{H}_{2} \mathrm{O}$ was added until no further reaction took place. Stirring for one $\mathrm{h}$ was followed by filtration over hyflo. The residuc was washed with warm ether $(2 x)$. The combined filtrates were washed with water. After drying $\left(\mathrm{MgSO}_{4}\right)$ the solvent was evaporated in vacuo, to yield pure 3 as an oil $(6.0 \mathrm{~g}$,

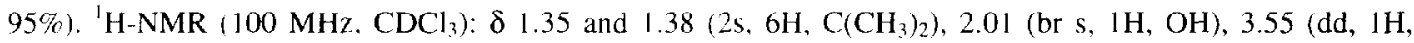
OCHHCHO $), 4.10(\mathrm{~m}, 3 \mathrm{H}, \mathrm{OCH} \underline{H C H O}$ and $\mathrm{CH}$ IR $\left(\mathrm{CCl}_{4}\right): v 3600,3450,2980,2940.2875,1380.1370 .1210,1060 \mathrm{~cm}^{-1}$.

( $2 S, 3 R)-3-/(4 R)-2.2-$ dimethyl-1,3-dioxolan-4-y//oxtran-2-yl/methanol (4a):

A suspension of finely powdered, activated molecular sieves (4A. $0.5 \mathrm{~g}$ ) in dry dichloromethane (20 ml) under nitrogen was cocled to -20$)^{\circ} \mathrm{C}$ and $\operatorname{Ti}(\mathrm{OPPr})_{4}(283 \mathrm{ml} .9 .5 \mathrm{mmol})$ and $\left.\mathrm{L}-1+\right)$-diethyl tartrate $(2.35 \mathrm{~g}, 11.4 \mathrm{mmol}$, dissolved in $3 \mathrm{ml}$ of dry dichloromethane) were successively added. The mixture was stirred (20 min) and allylic alcohol 3 ( $1.5 \mathrm{~g}, 9.5 \mathrm{mmol}$ dissolved in $10 \mathrm{ml}$ of dry dichloromethane) was added over five min. After stirring $(30 \mathrm{~min})$ a tert-butyl hydroperoxide solution in 1,2-dichloroethane $(5.40 \mathrm{ml}$ of a $3.4 \mathrm{M}$ solution, 2 equiv.) was added dropwise. The mixture was kept at $-20^{\prime \prime} \mathrm{C}$ overnight. The reaction was quenched by adding a solution of $3.4 \mathrm{~g}$ of $\mathrm{FeSO}_{4} .7 \mathrm{H}_{2} \mathrm{O}$ and $1.1 \mathrm{~g}$ of tartaric acid in $10 \mathrm{ml}$ of water at $0^{\circ} \mathrm{C}$. After stirring for 10 min the mixture was filtered over hyflo, the residue was washed with $25 \mathrm{ml}$ of dichloromethane (3x). The filtrate layers were separated and the aqueous layer was extracted with ether $(7 \mathrm{x})$. The combined organic layers were stirred at $0^{\circ} \mathrm{C}$ for one $\mathrm{h}$ with $5 \mathrm{ml}$ of a $30 \% \mathrm{NaOH}$ (w/v) solution in saturated brine ${ }^{3 b}$, after which the layers were separated and the aqueous layer was washed with ether $(3 \times)$. Drying of the combined organic layers $\left(\mathrm{MgSO}_{4}\right)$ and concentration gave $1.58 \mathrm{~g}$ of crude product. This was chromatographed (hexane/ethyl acetate $2: 1$ ), to give pure $4 \mathbf{a}$ as an oil $(1.16 \mathrm{~g}, 70 \%)$. $[\alpha]^{25} \mathrm{D}-20.5^{\circ}\left(\mathrm{c} 1.21, \mathrm{CHCl}_{3}\right):$ lit. $^{14}[\alpha]_{\mathrm{n}}-21.5^{\circ}$ (c $\left.0.77, \mathrm{CHCl}_{3}\right): \mathrm{lit}^{20}[\alpha]^{20} \mathrm{D}$ $-26^{\prime \prime}$ ( c 2.1, $\left.\mathrm{CH}_{2} \mathrm{Cl}_{2}\right) .{ }^{1} \mathrm{H}-\mathrm{NMR}\left(400 \mathrm{MHz}, \mathrm{CDCl}_{3}\right): \delta 1.37$ and $1.45\left(2 \mathrm{~s}, 6 \mathrm{H}, \mathrm{C}\left(\mathrm{CH}_{3}\right)_{2}\right), 2.40$ (br s, $\left.1 \mathrm{H}, \mathrm{OH}\right)$,

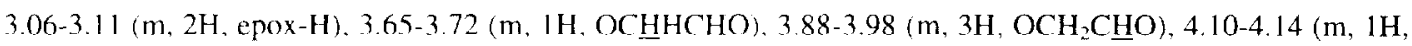
OCH $\underline{H C H O}) \mathrm{ppm} .{ }^{13} \mathrm{C}-\mathrm{NMR}(100 \mathrm{MHz}): \delta 25.2 .26 .4,55.2,57.1,61.0 .66 .7,75.2,109.8 \mathrm{ppm} . \mathrm{IR}\left(\mathrm{CCl}_{4}\right): \mathrm{v}$ $3600,3480,2990,2935,2875,1380,1370,1210,1060 \mathrm{~cm}^{\prime}$. MS (CI): m/e (\%) $175\left(12, \mathrm{M}^{+}+1\right), 159(96), 117$ (12, $\left.-\mathrm{CH}_{3} \mathrm{C}(\mathrm{O}) \mathrm{CH}_{3}\right) .101\left(15,-\mathrm{CHOCHCH}_{2} \mathrm{OH}\right), 99(41), 84(17), 69(33)$.

(2R,3R)-3-1(4R)-2,2-dimethyl-I.3-dioxolan-4-y/oxiran-2-y//carbaldehyde:

$\mathrm{CrO}_{3}(3.0 \mathrm{~g}, 33 \mathrm{mmol})$ was suspended in dry dichloromethane $(55 \mathrm{ml})$, followed by the addition of pyridine $14.80 \mathrm{ml}, 30 \mathrm{mmol})$. The mixture was stirred for 10 min after which epoxy alcohol 4a $(870 \mathrm{mg}, 5.0 \mathrm{mmol}$ dissolved in $6 \mathrm{~m}$ i of dry dichloromethanei was added. After $20 \mathrm{~min}$ the reaction was complete according to TLC 
and ether $(55 \mathrm{ml})$ was added, followed by stirring for $15 \mathrm{~min}$. The organic layer was decanted, the residue was washed with ether $(2 x)$ and with warm ether $(3 x)$. The combined organic layers were chromatographed (ether) over a $15 \mathrm{~cm}$ florisil column, giving after concentration crude aldehyde (622 $\mathrm{mg}, 73 \%$ ), which was immediately converted into acid 5a. ${ }^{1} \mathrm{H}-\mathrm{NMR}\left(100 \mathrm{MHz}, \mathrm{CDCl}_{3}\right), \delta 1.36$ and $1.42\left(2 \mathrm{~s}, 6 \mathrm{H}, \mathrm{C}\left(\mathrm{CH}_{3}\right)_{2}\right), 3.33-3.43(\mathrm{~m}, 2 \mathrm{H}$, epox-H), 3.85-4.11 (m, 3H, OC $\underline{H}_{2} \mathrm{CH}$ and $\left.\mathrm{OCH}_{2} \mathrm{CH}\right), 9.06(\mathrm{~d}, 1 \mathrm{H}, \mathrm{CHOCH}(\mathrm{O}), \mathrm{J} 6 \mathrm{~Hz}) \mathrm{ppm}$. IR $\left(\mathrm{CCl}_{4}\right): v$ $2990,2930,2890,1735,1380,1370,1070 \mathrm{~cm}^{-1}$.

$((2 R, 3 R)-3-((4 R)-2,2$-dimethyl-1,3-dioxelan-4-yl/oxiran-2-yl/carbox vlic acid (5a):

Pyridinium dichromate (PDC, $2.6 \mathrm{~g}, 7.2 \mathrm{mmol}$ ) was dissolved in dry DMF (3.5 ml). The mixture was stirred for $4 \mathrm{~min}$ after which the aldehyde ( $620 \mathrm{mg}, 3.6 \mathrm{mmol}$ dissolved in $4 \mathrm{ml}$ of dried DMF) was added quickly. Stirring was continued for $75 \mathrm{~min}$ and then ether $(20 \mathrm{ml})$ was added, followed by another $15 \mathrm{~min}$. of stirring. The organic solvent was decanted, the residue was washed with warm ether $(4 \mathrm{x})$. The combined organic solvents were filtered (hyflo) and concentrated until constant weight, yielding crude acid $\mathbf{5 a}(350 \mathrm{mg}, 52 \%)$. IR $\left(\mathrm{CCl}_{4}\right): \mathrm{v}$ $3500-2500,2980,2930,2880,1680,1380,1370 \mathrm{~cm}^{-1}$. For instability reasons the product was immediately converted into diazo ketone $6 \mathbf{a}$

1-f(2R,3R)-3-[(4R)-2,2-dimethyl-1,3-dioxolan-4-yl]oxiran-2-yl/-2-diazo-ethanone (6a):

Crude glycidic acid 5 a $(350 \mathrm{mg}, 1.86 \mathrm{mmol})$ was dissolved in dry ether $(30 \mathrm{ml})$ under nitrogen at $0^{\circ} \mathrm{C}$. Iso-butyl chloroformate $(276 \mu \mathrm{l}, 1.86 \mathrm{mmol}$ ) was added, followed by dried triethylamine (404 $\mu \mathrm{l}, 2.88 \mathrm{mmol})$. A white solid appeared, which was filtered off (under nitrogen) after one h of stirring. To the filtrate was added a $0.3 \mathrm{M}$ diazomethane solution in ether $(20 \mathrm{ml})$. After stirring for $2 \mathrm{~h}$ excess diazomethane was evaporated and the mixture was concentrated and chromalographed (hexantethyi acetate 3:2), to give pure diazo ketone 6a as an oil (252 mg, 64\%). ${ }^{1} \mathrm{H}-\mathrm{NMR}\left(100 \mathrm{MHz}, \mathrm{CDCl}_{3}\right): \delta 1.36$ and $1.43\left(2 \mathrm{~s}, 6 \mathrm{H}, \mathrm{C}\left(\mathrm{CH}_{3}\right)_{2}\right), 3.11$ (dd, $1 \mathrm{H}, \mathrm{CHCHC}(\mathrm{O})$, J $5 \mathrm{~Hz}, 1.7 \mathrm{~Hz}), 3.44(\mathrm{~d}, 1 \mathrm{H}, \mathrm{CHC} \underline{\mathrm{HC}}(\mathrm{O}), \mathrm{J} 1.7 \mathrm{~Hz}), 3.84-4.16\left(\mathrm{~m}, 3 \mathrm{H}, \mathrm{C}_{2} \mathrm{CH}\right.$ and $\left.\mathrm{CH}_{2} \mathrm{C} \underline{\mathrm{H}}\right), 5.53(\mathrm{~s}, 1 \mathrm{H}$, $\left.\mathrm{CHN}_{2}\right)$ ppm. IR $\left(\mathrm{CCl}_{4}\right): v 3120,2980,2930,2880,2105,1645,1380,1370 \mathrm{~cm}^{-1}$.

Methyl E-4-[(4R)-2,2-dimethvl-1,3-dioxolan-4-vl]-4-(R)-hydroxy-but-2-enoate (7a):

Diazo ketone $6 \mathrm{a}(100 \mathrm{mg}, 0.47 \mathrm{mmol}$. dissolved in $45 \mathrm{ml}$ of absolute methanol) was irradiated under nitrogen at $300 \mathrm{~nm}$. The progress of the reaction was monitored by IR. After $75 \mathrm{~min}$ the diazo peak had disappeared. Concentration of the mixture gave $92 \mathrm{mg}$ of crude product, which was chromatographed (hexane/ethyl acetate 2:1), yielding unsaturated ester $7 \mathbf{a}$ as a solid $(53 \mathrm{mg}, 52 \%)$. M.p. (pentane) $74-74.5^{\circ} \mathrm{C}$. ${ }^{1} \mathrm{H}-\mathrm{NMR}(100 \mathrm{MHz}$, $\left.\mathrm{CDCl}_{3}\right): \delta 1.37$ and $1.46\left(2 \mathrm{~S}, 6 \mathrm{H}, \mathrm{C}\left(\mathrm{CH}_{3}\right)_{2}\right), 2.60(\mathrm{~d}, 1 \mathrm{H}, \mathrm{OH}, \mathrm{J} 7 \mathrm{~Hz}), 3.75\left(\mathrm{~s}, 3 \mathrm{H}, \mathrm{OCH}_{3}\right), 3.83-4.30(\mathrm{~m}, 4 \mathrm{H}$, $\mathrm{OCH}_{2} \mathrm{CH}, \mathrm{CH}_{2} \mathrm{CHO}$ and $\mathrm{CH}(\mathrm{OH}) \mathrm{C}$ ), 6.17 (dd, $1 \mathrm{H}, \mathrm{C}=\mathrm{CHC}(\mathrm{O}), \mathrm{J} 16 \mathrm{~Hz}$ and $\left.1.6 \mathrm{~Hz}\right), 6.88$ (dd, $1 \mathrm{H}$, $\mathrm{CH}=\mathrm{CHC}(\mathrm{O}), \mathrm{J} 16 \mathrm{~Hz}$ and $4.5 \mathrm{~Hz}) \mathrm{ppm} . \mathrm{IR}\left(\mathrm{CCl}_{4}\right): \vee 3600-3300,2980,2940,2880,1725,1660,1380,1370$ $\mathrm{cm}^{-1}$. MS (Cl): m/e (\%) $217\left(30, \mathrm{M}^{+}+1\right), 201\left(39,{ }^{+} \mathrm{CH}_{3}\right), 199\left(10,-\mathrm{H}_{2} \mathrm{O}\right), 185(3), 169(8), 159\left(28,-\mathrm{CO}_{2} \mathrm{Me}\right)$, $141\left(19,-\mathrm{CO}_{2} \mathrm{Me},-\mathrm{H}_{2} \mathrm{O}\right), 127\left(32,-\mathrm{H}_{2} \mathrm{O},-\mathrm{CHCO}_{2} \mathrm{Me}\right), 101\left(100,-\mathrm{CH}(\mathrm{OH}) \mathrm{CH}=\mathrm{CHCO}_{2} \mathrm{Me}\right)$. El/HRMS: $m / e$ calcd. for $\mathrm{C}_{10} \mathrm{H}_{16} \mathrm{O}_{5} 216.09977$, found $216.09962 \pm 0.00082$ a.m.u. Calcd. for $\mathrm{C}_{10} \mathrm{H}_{16} \mathrm{O}_{5}$ : C 55.55, $\mathrm{H} 7.46 \%$, found $\mathrm{C} 54.91, \mathrm{H} 7.26 \%$. 
$\{(2 R, 3 S)-3-[(4 R)-2,2-$ dimethyl-1,3-dioxolan-4-y/)oxiran-2-yl/methanol (4b):

Epoxy alcohol 4b was prepared following the procedure for epoxy alcohol 4a, using D-(-)-diethyl tartrate as chiral inductor, as crude product. No extensive extractions were performed (cf. compound $4 \mathbf{a}$ ), consequently the yield was relatively low. After chromatography (hexane/ethyl acetate $2: 1$ ) pure 4a was obtained as an oil (34\% yield). $[\alpha]^{25}+35.8^{\circ}$ (c $\left.1.07, \mathrm{CHCl}_{3}\right)$; lit. ${ }^{14}[\alpha]_{\mathrm{D}}+38^{\circ}$ (c $\left.1.45, \mathrm{CHCl}_{3}\right) ;$ lit. ${ }^{20}[\alpha]^{20}{ }_{\mathrm{D}}+29^{\circ}$ (c $\left.3.2, \mathrm{CH}_{2} \mathrm{Cl}_{2}\right) .{ }^{1} \mathrm{H}-$ NMR (100 MHz, $\left.\mathrm{CDCl}_{3}\right): \delta 1.37$ and $1.45\left(25,6 \mathrm{H}, \mathrm{C}\left(\mathrm{CH}_{3}\right)_{2}\right), 1.62(\mathrm{~s}, 1 \mathrm{H}, \mathrm{OH}), 3.05-3.20(\mathrm{~m}, 2 \mathrm{H}$, epox-H), $3.50-4.20\left(\mathrm{~m}, 5 \mathrm{H}, \mathrm{OCH}_{2} \mathrm{CH}, \mathrm{CH}_{2} \mathrm{CHO}\right.$ and $\left.\mathrm{CH}_{2} \mathrm{OH}\right) \mathrm{ppm}$. IR $\left(\mathrm{CCl}_{4}\right): v 3600,3480,2990,2870,1380,1370$, 1210, $1060 \mathrm{~cm}^{-1}$. MS (CI): m/e (\%) $175\left(6, \mathrm{M}^{+}+1\right), 159(96), 117 \quad\left(14,-\mathrm{CH}_{3} \mathrm{C}(\mathrm{O}) \mathrm{CH}_{3}\right), 101 \quad(29$, $\left.-\mathrm{CHOCHCH}_{2} \mathrm{OH}\right), 99(50), 69(37)$.

(2S,3S)-3-[(4R)-2.2-dimethyl-1,3-dioxalan-4-yl]oxiran-2-yl/carbaldehyde:

This aldehyde was prepared following the method for the $(2 \mathrm{R}, 3 \mathrm{~S})$-diastereomer. Starting with epoxy alcohol $\mathbf{4 b}$ (450 my. 2.6 Innol), crude aldehyde was synthesized as an oil (289 mg, 65\%). ${ }^{1} \mathrm{H}-\mathrm{NMR}\left(100 \mathrm{MHz}, \mathrm{CDCl}_{3}\right): \delta$ 1.35 and $1.42\left(2 \mathrm{~s}, 6 \mathrm{H}, \mathrm{C}\left(\mathrm{CH}_{3}\right)_{2}\right), 3.25-3.35\left(\mathrm{~m}, 2 \mathrm{H}\right.$, epox-H), 3.86-4.21 (m, 3H, $\mathrm{OCH}_{2} \mathrm{CH}$ and $\left.\mathrm{CH}_{2} \mathrm{CH}\right), 9.06(\mathrm{~d}$, $1 \mathrm{H}, \mathrm{CH}(\mathrm{O}), \mathrm{J} 6 \mathrm{~Hz}) \mathrm{ppm}$. IR $\left(\mathrm{CCl}_{4}\right): v 2990,2935,2880,1735,1380,1370,1065 \mathrm{~cm}^{-1}$. This aldehyde was immediately converted into acid $\mathbf{5 b}$.

$\{(2 S, 3 S)-3-[(4 R)-2,2-$ dimethyl-1,3-dioxolan-4-yl]oxiran-2-yl\}carboxylic acid (5b):

Following the procedure for the preparation of glycidic acid $\mathbf{5 a}$, compound $\mathbf{5 b}$ was synthesized as an oil (80\% yield). IR $\left(\mathrm{CCl}_{4}\right):$ v 3600-2500,2980, 2930, 2870,1680,1380, $1370 \mathrm{~cm}^{-1}$. The product was immediately converted into diazoketone $\mathbf{6 b}$.

1-\{(2S,3R)-3-[(4R)-2,2-dimethyl-1,3-dioxolan-4-yl]oxiran-2-yl)-2-diazo-ethanone (6b):

Starting with glycidic acid $\mathbf{5 b}(300 \mathrm{mg}, 1.60 \mathrm{mmol}$ ) and following the procedure for compound $\mathbf{6 a}$, pure epoxy diazo ketone $\mathbf{6 b}$ (chromatographed with hexane/ethyl acetate 2:1) was obtained as an oil (80 $\mathrm{mg}, 25 \%)$. ${ }^{1} \mathrm{H}-\mathrm{NMR}\left(100 \mathrm{MHz}, \mathrm{CDCl}_{3}\right): \delta 1.36$ and $1.45\left(2 \mathrm{~s}, 6 \mathrm{H}, \mathrm{C}\left(\mathrm{CH}_{3}\right)_{2}\right), 3.09$ (dd, $1 \mathrm{H}, \mathrm{CHCHC}(\mathrm{O}), \mathrm{J} 4.4 \mathrm{~Hz}$ and 1.8 $\mathrm{Hz}), 3.35(\mathrm{~d}, 1 \mathrm{H}, \mathrm{CHC} \underline{\mathrm{HC}}(\mathrm{O}), \mathrm{J} 1.8 \mathrm{~Hz}), 3.77-4.13\left(\mathrm{~m}, 3 \mathrm{H}, \mathrm{OC}_{2} \mathrm{CH}_{\text {and }} \mathrm{CH}_{2} \mathrm{C} \underline{\mathrm{H}}\right.$ ), 5.50 (s, $1 \mathrm{H}, \mathrm{CHN}_{2}$ ) ppm. IR $\left(\mathrm{CCl}_{4}\right): \vee 3120,2980,2940,2880,2105,1645,1380.1370 \mathrm{~cm}^{-1}$.

Methyl E-4-[(4R)-2,2-dimethyl-J,3-dioxolan-4-yl]-4-(S)-hydroxy-but-2-enoate (7b):

Starting with diazoketone $\mathbf{6 b}(80 \mathrm{mg}, 0.38 \mathrm{mmol})$ and following the procedure for compound $7 \mathbf{a}$, pure ester $7 \mathbf{b}$ was obtained as an oil (52 mg, 64\%) after chromatography (hexane/ethyl acetate $2: 1$ ). ${ }^{1} \mathrm{H}-\mathrm{NMR}$ (400 $\mathrm{MHz}$, $\left.\mathrm{CDCl}_{3}\right): \delta 1.37$ and $1.46\left(2 \mathrm{~s}, 6 \mathrm{H}, \mathrm{C}\left(\mathrm{CH}_{3}\right)_{2}\right), 2.45$ (br s, $\left.1 \mathrm{H}, \mathrm{OH}\right), 3.76\left(\mathrm{~s}, 3 \mathrm{H}, \mathrm{OCH}_{3}\right), 3.89(\mathrm{dd}, 1 \mathrm{H}, \mathrm{OCHHCHO}$, J $8.5 \mathrm{~Hz}$ and $6.2 \mathrm{~Hz}), 3.95(\mathrm{dd}, \mathrm{lH}, \mathrm{OCH} H C H O, \mathrm{~J} 8.5 \mathrm{~Hz}$ and $6.6 \mathrm{~Hz}), 4.16\left(\mathrm{~m}, 1 \mathrm{H}, \mathrm{CH}_{2} \mathrm{CHO}\right), 4.48(\mathrm{~m}, 1 \mathrm{H}$, $\mathrm{C} \underline{H}(\mathrm{OH}) \mathrm{C}), 6.19(\mathrm{dd}, 1 \mathrm{H}, \mathrm{CH}=\mathrm{C} \underline{\mathrm{HC}}(\mathrm{O}), \mathrm{J} 15.7 \mathrm{~Hz}$ and $1.9 \mathrm{~Hz}), 6.92(\mathrm{dd}, 1 \mathrm{H}, \mathrm{CH}=\mathrm{CHC}(\mathrm{O}), \mathrm{J} 15.7 \mathrm{~Hz}$ and 4.1 $\mathrm{Hz}) \mathrm{ppm}$. IR $\left(\mathrm{CCl}_{4}\right): \vee 3600-3300,2990,2940,2880,1725,1665,1380,1370 \mathrm{~cm}^{-1}$. MS (CI): m/e (\%) $217(27$, $\left.\mathrm{M}^{+}+1\right), 201\left(33,-\mathrm{CH}_{3}\right), 199\left(8,-\mathrm{H}_{2} \mathrm{O}\right), 185(12), 169(11), 159\left(24,-\mathrm{CO}_{2} \mathrm{Me}\right), 141\left(11,-\mathrm{CO}_{2} \mathrm{Me},-\mathrm{H}_{2} \mathrm{O}\right), 127$ 
(34, $\left.-\mathrm{H}_{2} \mathrm{O},-\mathrm{CHCO}_{2} \mathrm{Me}\right), 101\left(100,-\mathrm{CH}(\mathrm{OH}) \mathrm{CH}=\mathrm{CHCO}_{2} \mathrm{Me}\right)$. El/HRMS: $m / e$ calcd. for $\mathrm{C}_{10} \mathrm{H}_{16} \mathrm{O}_{5} 216.09977$, found $216.09983 \pm 0.00082$ a.m.u.

(2S,4S)-2-Phenyl-1,3-dioxane-4-carbaldehyde:

To a stirred solution of freshly distilled oxalyl chloride $(3.84 \mathrm{ml}, 44 \mathrm{mmol})$ in dry dichloromethane $(100 \mathrm{ml})$ dimethyl sulfoxide $\left(6.9 \mathrm{ml}, 80 \mathrm{mmol}\right.$ dissolved in $15 \mathrm{ml}$ of dry dichloromethane) was added at $-78^{\circ} \mathrm{C}$ under nitrogen. The white suspension was stirred for $10 \mathrm{~min}$, after which alcohol $\underline{8}$ was added $(7.68 \mathrm{~g}, 39.6 \mathrm{mmol})$, to give a clear solution. Stirring for $40 \mathrm{~min}$ was followed by addition of diisopropylethylamine $(35 \mathrm{ml}, 195$ $\mathrm{mmol}$ ). The mixture was allowed to reach room temperature, after which it was washed with water. The organic layer was extracted successively with $1 \% \mathrm{HCl}, 5 \% \mathrm{Na}_{2} \mathrm{CO}_{3}$ and brine. After drying $\left(\mathrm{MgSO}_{4}\right)$ the mixture was concentrated in vacuo to give the crude aldehyde $(7.12 \mathrm{~g}, 94 \%)$, which was reacted further without purification. ${ }^{1} \mathrm{H}-\mathrm{NMR}\left(100 \mathrm{MH} z, \mathrm{CDCl}_{3}\right): \delta \quad 1.60-2.10\left(\mathrm{II}, 2 \mathrm{H}, \mathrm{OCH}_{2} \mathrm{CH}_{2} \mathrm{CH}\right), 3.60-4.35\left(\mathrm{~m}, 3 \mathrm{H}, \mathrm{OCH}_{2} \mathrm{CH}_{2} \mathrm{CH}_{\text {and }}\right.$ $\mathrm{OCH}_{2} \mathrm{CH}_{2} \mathrm{CH}$ ), $5.60(\mathrm{~s}, 1 \mathrm{H}, \mathrm{CHPh}), 7.30-7.64(\mathrm{~m}, 5 \mathrm{H}, \mathrm{Ph}), 9.72(\mathrm{~s}, 1 \mathrm{H}, \mathrm{CHC}(\mathrm{O}) \underline{\mathrm{H}}) \mathrm{ppm} . \mathrm{IR}\left(\mathrm{CCl}_{4}\right): v 3090$, $3065,3040,2970,2930,2850,2820,1740,1370 \mathrm{~cm}^{-1}$.

Ethyl E-3-[(2S,4S)-2-phenyl-1,3-dioxan-4-yl]prop-2-enoate:

To a suspension of oven-dried $\mathrm{LiCl}(1.855 \mathrm{~g}, 43.75 \mathrm{mmol})$ in dry acetonitrile $(200 \mathrm{ml})$ under nitrogen and at room temperature was added triethyl phosphonoacetate $(9.8 \mathrm{~g}, 43.74 \mathrm{mmol})$ and diisopropylethylamine $(6.33$ $\mathrm{ml}, 36.46 \mathrm{mmol}$ ). Stirring for $5 \mathrm{~min}$ was followed by the addition of crude aldehyde (described above, $7.0 \mathrm{~g}$, $36.36 \mathrm{mmol}$ ) in dry $\mathrm{CH}_{3} \mathrm{CN}(50 \mathrm{ml})$. The mixture was stirred for $2 \mathrm{~h}$ (progress of reaction monitored by TLC), after which water was added until all salts had dissolved. Acetonitrile was evaporated and the residue was taken up in water and washed with ether $(3 \mathrm{x})$. The combined ethereal layers were dried $\left(\mathrm{MgSO}_{4}\right)$ and concentrated in vacuo to yield a yellow solid as crude product $(11.12 \mathrm{~g})$. This was chromatographed (hexane/ethyl acetate $4: 1)$, giving pure trans ester as a solid $(6.12 \mathrm{~g}, 65 \%)$. M.p. (pentane) $67.5-68^{\circ} \mathrm{C} .[\alpha]^{25} \mathrm{D}-22.6^{\circ}\left(\mathrm{c} 1.5, \mathrm{CHCl}_{3}\right)$, lit. $^{25 \mathrm{a}}$ $\left.[\alpha]^{23}{ }_{D}-23.8^{\circ}(c) 1.8, \mathrm{CHCl}_{3}\right)$. 'H-NMR $\left(100 \mathrm{MHz} \mathrm{CDCl}_{3}\right): \delta 1.29\left(\mathrm{t}, 3 \mathrm{H}, \mathrm{CH}_{3}, \mathrm{~J} 7.2 \mathrm{~Hz}\right), 1.58-2.12(\mathrm{~m}, 2 \mathrm{H}$, $\left.\mathrm{OCH}_{2} \mathrm{CH}_{2} \mathrm{CH}\right), 3.86-4.40\left(\mathrm{~m}, 2 \mathrm{H}, \mathrm{OCH}_{2} \mathrm{CH}_{2} \mathrm{CH}\right), 4.41-4.67(\mathrm{~m}, 1 \mathrm{H}, \mathrm{CHOCH}=\mathrm{C}), 4.21\left(\mathrm{q}, 2 \mathrm{H}, \mathrm{OCH}_{2} \mathrm{CH}_{3}, \mathrm{~J} 7.1\right.$ $\mathrm{Hz}), 5.59(\mathrm{~s}, 1 \mathrm{H}, \mathrm{CHPh}), 6.13(\mathrm{dd}, 1 \mathrm{H} . \mathrm{C}=\mathrm{CHC}(\mathrm{O}), \mathrm{J} 15.7 \mathrm{~Hz}$ and $1.8 \mathrm{~Hz}), 6.97(\mathrm{dd}, 1 \mathrm{H}, \mathrm{C} \underline{\mathrm{H}}=\mathrm{CHC}(\mathrm{O}), \mathrm{J} 15.7$ $\mathrm{Hz}$ and $4.1 \mathrm{~Hz}), 7.29-7.58(\mathrm{~m} .5 \mathrm{H}, \mathrm{Ph}) \mathrm{ppm}$. IR $\left(\mathrm{CCl}_{4}\right): v$ 3090, 3065, 3040, 2980, 2960, 2920, 2850, 1720. 1660, 1540, $1300 \mathrm{~cm}^{-1}$. MS (CI): m/e (\%) 263 (39, $\left.\mathrm{M}^{+}+1\right), 217$ (42, -EtOH), 157 (61, - PhC(O)H), 139 (44, $\left.-\mathrm{PhC}(\mathrm{O}) \mathrm{H},-\mathrm{H}_{2} \mathrm{O}\right), 127$ (58, - $\left.\mathrm{EtOH},-\mathrm{PhCH}\right), 111(67,-\mathrm{PhC}(\mathrm{O}) \mathrm{H},-\mathrm{EtOH}), 107\left(62, \mathrm{PhC}(\mathrm{O}) \mathrm{H}^{+}+1\right), 106(46$, $\left.\mathrm{PhC}(\mathrm{O}) \mathrm{H}^{+}\right), 105\left(100, \mathrm{PhC}(\mathrm{O})^{+}\right), 94(57), 83(47), 67(55)$. Calcd. for $\mathrm{C}_{15} \mathrm{H}_{18} \mathrm{O}_{4}: \mathrm{C} 68.69, \mathrm{H} \mathrm{6.92 \%}$, found $\mathrm{C}$ 67.72, H $6.81 \%$.

\section{E-3-\{(2S.4S)-2-phenyl-1.3-dioxan-4-yl/prop-2-en-ol (9):}

Allylic alcohol 9 was synthesized from the $\alpha, \beta$-unsaturated ester described above, following the procedure for the preparation of allylic alcohol 3. Starting with the ester $(3.0 \mathrm{~g}, 11.45 \mathrm{mmol})$ trans-alcohol 9, purified by chromatography (hexane/ethyl acetate $3: 1)$, was prepared as an oil (1.88 g, 75\%). 'H-NMR (100 MHz, $\mathrm{CDCl}_{3}$ ): $\delta$ 1.41-1.60 (br d, $1 \mathrm{H}, \mathrm{OCH}_{2} \mathrm{CHH}, \mathrm{J} 13 \mathrm{~Hz}$ ), 1.93 (dq. $1 \mathrm{H} . \mathrm{OCH}_{2} \mathrm{CHHCH}, \mathrm{J} 11 \mathrm{~Hz}$ and $4.9 \mathrm{~Hz}$ ), 2.76 (br s, $1 \mathrm{H}$, $\mathrm{OH}), 3.83-4.27\left(\mathrm{~m}, 4 \mathrm{H}, \mathrm{OCH}_{2} \mathrm{CH}_{2} \mathrm{CHO}\right.$ and $\left.\mathrm{C}=\mathrm{CC} \underline{H}_{2} \mathrm{OH}\right), 4.28-4.47\left(\mathrm{~m}, 1 \mathrm{H}, \mathrm{OCH}_{2} \mathrm{CHCHO}\right), 5.55(\mathrm{~s}, 1 \mathrm{H}$, 
$\mathrm{CHPh}), 5.81-5.91(\mathrm{~m}, 2 \mathrm{H}, \mathrm{C} \underline{\mathrm{H}}=\mathrm{C} \underline{\mathrm{H}}), 7.27-7.57(\mathrm{~m}, 5 \mathrm{H}, \mathrm{Ph}) \mathrm{ppm}$. IR $\left(\mathrm{CCl}_{4}\right): \vee 3610,3440,3190,3160,3140$, 2980, 2950, 2920, 2850, $1550 \mathrm{~cm}^{-1}$. MS (CI): m/e (\%) 22] (41, $\left.\mathrm{M}^{+}+1\right), 203\left(19,-\mathrm{H}_{2} \mathrm{O}\right), 163(6$, $\left.-\mathrm{CH}=\mathrm{CHCH}_{2} \mathrm{OH}\right), 149(15), 134$ (19), $115(21), 107$ (35, $\left.\mathrm{PhC}(\mathrm{O}) \mathrm{H}^{+}+1\right), 106\left(20, \mathrm{PhC}(\mathrm{O}) \mathrm{H}^{+}\right), 105(45), 97$ $\left(100,-\mathrm{PhC}(\mathrm{O}) \mathrm{H},-\mathrm{H}_{2} \mathrm{O}\right), 91$ (26), 80 (39), 69 (27), 49 (29). EL/HRMS: m/e calcd. for $\mathrm{C}_{13} \mathrm{H}_{16} \mathrm{O}_{3} 220.10994$, found $220.10990 \pm 0.00088$ a.m.u.

$\{(2 S, 3 R)-3-[(2 S, 4 S)-2-p h e n y l-1,3-d i o x a n-4-y l]$ oxiran-2-vl/methanol (10a):

Compound 10a was prepared following the procedure for epoxy alcohol $\mathbf{4 a}$. Starting with allylic alcohol 9 (950 $\mathrm{mg}, 4.3 \mathrm{mmol}$ ) and using L-(+)-DET as chiral inductor, crude epoxide was obtained as a white solid (965 $\mathrm{mg}$ ), which was recrystallized from ether at $-20^{\circ} \mathrm{C}$. yielding epoxy alcohol 10a $(568 \mathrm{mg}, 56 \%)$. M.p. $84-84.5^{\circ} \mathrm{C}$. $[\alpha]^{25}{ }_{\mathrm{D}}-8.85^{\circ}\left(\mathrm{c} 1.17, \mathrm{CHCl}_{3}\right)$. ${ }^{\mathrm{H}} \mathrm{H}-\mathrm{NMR}\left(100 \mathrm{MHz}_{2} \mathrm{CDCl}_{3}\right): \delta 1.50-1.77\left(\mathrm{~m}, 2 \mathrm{H}, \mathrm{CH}_{2} \mathrm{CHHCHO}\right.$ and $\left.\mathrm{OH}\right), 2.01$ (dq, $1 \mathrm{H}, \mathrm{CH}_{2} \mathrm{CH}$ HCHO, $\mathrm{J} 11.5 \mathrm{~Hz}$ and $4.9 \mathrm{~Hz}$ ), 3.15 (dd, $1 \mathrm{H}, \mathrm{CHOCHOCH}_{2} \mathrm{OH}, \mathrm{J} 4.3 \mathrm{~Hz}$ and $2.0 \mathrm{~Hz}$ ), 3.25 (dt. 1H. CHOCHOCH${ }_{2} \mathrm{OH}, \mathrm{J} 6.0 \mathrm{~Hz}$ and $2.0 \mathrm{~Hz}$ ), 3.65 (ddd, $1 \mathrm{H}, \mathrm{CHOCHHOH}, \mathrm{J} 12 \mathrm{~Hz}, 7.5 \mathrm{~Hz}$ and $4.0 \mathrm{~Hz}$ ), 3.80$4.12\left(\mathrm{~m}, 3 \mathrm{H} \mathrm{CH} \mathrm{H}_{2} \mathrm{CH}_{2} \mathrm{CHO}\right.$ and $\mathrm{CHOCH} \underline{\mathrm{HOH}}$ ), 4.33 (ddd, $1 \mathrm{H}, \mathrm{CH}_{2} \mathrm{CH}_{2} \mathrm{CHOCHO}, \mathrm{J} 11.5 \mathrm{~Hz}, 6.0 \mathrm{~Hz}$ and 1.6 $\mathrm{Hz}), 5.50(\mathrm{~s}, 1 \mathrm{H}, \mathrm{CHPh}), 7.31-7.50(\mathrm{~m}, 5 \mathrm{H}, \mathrm{Ph}) \mathrm{ppm}$. IR $\left(\mathrm{CCl}_{4}\right): \vee 3500,2960,2870.1120 \mathrm{~cm}^{-1}$. MS (CI): m/e (\%) $237\left(4, \mathrm{M}^{+}+1\right), 236\left(24, \mathrm{M}^{+}\right), 235\left(47, \mathrm{M}^{+}-1\right), 163\left(67,-\mathrm{CHOCHCH}_{2} \mathrm{OH}\right), 131(13,-\mathrm{PhC}(\mathrm{O}) \mathrm{H}), 113(55$, -PhC(O)H, -H2O). 107 (81. $\left.\mathrm{PhC}(\mathrm{O}) \mathrm{H}^{+}+1\right) .106$ (32. $\left.\left.\mathrm{PhC}^{\circ} \mathrm{O}\right) \mathrm{H}^{+}\right), 105\left(\mathrm{PhC}(\mathrm{O})^{+}\right), 95(24), 91(50), 87(72), 83$ (61), 79 (52). 77 (46), 71 (73), 67 (41), 55 (50), 41 (100). EI/HRMS: m/e calcd. for $\mathrm{C}_{13} \mathrm{H}_{16} \mathrm{O}_{4} 236.1049$, found $236.10496 \pm 0.00046$ a.m.u. Calcd. for $\mathrm{C}_{13} \mathrm{H}_{16} \mathrm{O}_{4}: \mathrm{C} 66.09, \mathrm{H} 6.83 \%$, found $\mathrm{C} 66.66, \mathrm{H} 6.92 \%$

$\{(2 R, 3 R)-3-[(2 S, 4 S)-2-p h e n y-1,3-$ dioxan-4-ylloxiran-2-yl/carboxylic acid (11a):

Glycidic acid 11a was synthesized in a two-step procedure from epoxy alcohol 10a via the aldehyde. Starting with the alcohol $(500 \mathrm{mg}, 2.12 \mathrm{mmol})$ and following the procedure for the synthesis of $(2 S, 4 S)$-2-phenyl-1,3dioxan-4-carbaldehyde (vide supra), crude aldehyde was prepared as an oil (623 $\mathrm{ng}$ ). 'H-NMR (100 MHz, $\mathrm{CDCl}_{3}$ ): $\delta 1.59$ (br d. $1 \mathrm{H}, \mathrm{CH}_{2} \mathrm{CHHOCH}, \mathrm{J} 13.1 \mathrm{~Hz}$. $1.99\left(\mathrm{dq}, 1 \mathrm{H}, \mathrm{CH}_{2} \mathrm{CHHOCH}, \mathrm{J} 11.4 \mathrm{~Hz}\right.$ and $5.2 \mathrm{~Hz}$ ), 3.35 (dd. $1 \mathrm{H}$, epox-H, J $3.9 \mathrm{~Hz}$ and $1.9 \mathrm{~Hz}$ ), $3.47(\mathrm{dd}, 1 \mathrm{H}$, epox-H, J $6.0 \mathrm{~Hz}$ and $1.9 \mathrm{~Hz}), 3.81-4.13(\mathrm{~m}, 2 \mathrm{H}$, $\mathrm{OCH}_{2} \mathrm{CH}_{2} \mathrm{CH}$ ). 4.31 (ddd. $1 \mathrm{H}, \mathrm{CH}_{2} \mathrm{CHOCHO}, \mathrm{J} 11.6 \mathrm{~Hz}, 5.2 \mathrm{~Hz}$ and $1.5 \mathrm{~Hz}$ ), 5.55 (s, $1 \mathrm{H}, \mathrm{CHPh}$ ), 7.20-7.53 $(\mathrm{m}, 5 \mathrm{H}, \mathrm{Ph}) .9 .05$ (d. 1H, CHO, J $6.0 \mathrm{~Hz}) \mathrm{ppm}$. IR $\left(\mathrm{CCl}_{4}\right): v 3090,3070,3040,2980,2960,2920,2850,2820$, $1735,1110,700 \mathrm{~cm}^{-1}$. The aldehyde was immediately dissolved in a mixture of tert-hutyl alcohol $(40 \mathrm{ml})$, 2-methyl-2-butene $(12 \mathrm{ml})$ and dichloromethane $(3 \mathrm{ml}$. to raise the solubility of the aldehyde), to which a solution of $\mathrm{NaClO}_{2}(1.7 \mathrm{~g} .18 .8 \mathrm{mmol})$ and $\mathrm{NaH}_{2} \mathrm{PO}_{4}(1.7 \mathrm{~g} .14 .2 \mathrm{mmol})$ in water $(17 \mathrm{ml})$ was added. The slightly yellow mixture was stirred overnight, after which the volatile solvents were evaporated. Acidification to $\mathrm{pH} 5$, followed by extraction with ether $(4 \mathrm{x})$, drying of the combined cthercal layers $\left(\mathrm{MgSO}_{4}\right)$ and concentration in vacuo yielded crude glycidic acid $11 \mathbf{a}(529 \mathrm{mg})$, which was immediately converted into diazo ketone 12a. 'H-NMR (100 MHz, CDCl 3 ): $\delta 1.49-1.74\left(\mathrm{~m}, 1 \mathrm{H}, \mathrm{CH}_{2} \mathrm{CH} H C H O\right), 1.80-2.23\left(\mathrm{~m}, 1 \mathrm{H} . \mathrm{CH}_{2} \mathrm{CH} \underline{\mathrm{HCHO}}\right.$ ), 3.36 (dd, 1H. epox-H, J $3.9 \mathrm{~Hz}$ and $1.8 \mathrm{~Hz}), 3.58(\mathrm{~d} .1 \mathrm{H}$, epox-H, J $1.8 \mathrm{~Hz}), 3.80-4.12\left(\mathrm{~m}, 2 \mathrm{H}, \mathrm{OCH}_{2} \mathrm{CH}_{2} \mathrm{CHO}\right), 4.22-$ $4.55\left(\mathrm{~m}, 1 \mathrm{H}, \mathrm{CH}_{2} \mathrm{CH}_{2} \mathrm{CHO}\right), 5.49$ (s, 1H, CHPh). 7.30-7.65 (m. 5H, Ph) ppm. IR $\left(\mathrm{CCl}_{4}\right): v$ 3600-2500, 2920, $2860.1725 \mathrm{~cm}^{-1}$. 
1-/(2R,3R)-3-[(2S.4S)-2-pheny-1,3-dioxan-4-yl]oxiran-2-y//-2-diazo-ethanone (12a):

Starting with crude acid 11 a (530 mg, $2.12 \mathrm{mmol}$ ) and following the procedure for the synthesis of diazoketone 5a. crude product was synthesized $(475 \mathrm{mg}$ ). This was chromatographed (hexane/ethyl acetate 1:1), yielding compound 12a as an oil ( $170 \mathrm{mg}, 30 \%$ ). The low yield is due to decomposition on the silica gel column, as was indicated by the isolation of benzaldehyde. ${ }^{1} \mathrm{H}-\mathrm{NMR}\left(100 \mathrm{MH}, \mathrm{CDCl}_{3}\right): \delta 1.52$ (br d, IH, $\mathrm{CH}_{2} \mathrm{CHHCHO}, \mathrm{J}$ 13.1 Hz), 1.70-2.20 (m, 1H, CH2 CHHCHO), 3.20 (dd. $1 \mathrm{H}$, epox-H. J $3.3 \mathrm{~Hz}$ and $2.0 \mathrm{~Hz}), 3.54$ (d, 1H, epox-H, J $2.0 \mathrm{~Hz}$ ), 3.92-4.18 (m, $2 \mathrm{H}, \mathrm{OCH}_{2} \mathrm{CH}_{2} \mathrm{CHO}$, 4.30 (ddd, $1 \mathrm{H}, \mathrm{CH}_{2} \mathrm{CH}_{2} \mathrm{CHO}, \mathrm{J} 10.5 \mathrm{~Hz}, 5.2 \mathrm{~Hz}$ and $1.3 \mathrm{~Hz}$ ), $5.49\left(\mathrm{~s}, 2 \mathrm{H}, \mathrm{CHN}_{2}\right.$ and $\left.\mathrm{CHPh}\right), 7.31-7.53\left(\mathrm{~m}, 5 \mathrm{H}\right.$. Ph) ppm. IR $\left(\mathrm{CCl}_{4}\right): v 3120,3060,2960,2920,2850,2110$, 1645, $1110,910,700 \mathrm{~cm}^{-1}$. MS (CI): $m / e(\%) 275\left(1, \mathrm{M}^{+}+1\right), 273\left(23, \mathrm{M}^{+}-1\right), 197(5), 163(35), 131(11), 124$ (7), $107\left(22, \mathrm{PhC}\left(\mathrm{O}_{1} \mathrm{H}^{+}+1\right), 106\left(15, \mathrm{PhC}(\mathrm{O}) \mathrm{H}^{+}\right), 105\left(100, \mathrm{PhC}(\mathrm{O})^{+}-1\right), 91\right.$ (32), 83 (22), 77 (19), 69 (23), 55 (24), 43 (23), 41 (41). EI/HRMS: $m / e$ calcd. for $\mathrm{C}_{14} \mathrm{H}_{14} \mathrm{~N}_{2} \mathrm{O}_{4} 274.09536$, found $274.09523 \pm 0.00081$ a.m.u.

Ethyl E-4-[(2S.4S)-2-pheny-1.3-dioxan-4-y/]-4-(R)-h)droxy-but-2-enoate (13a):

Following the procedure for the synthesis of unsaturated ester 7 a and starting with epoxy diazo ketone $12 a$ ( 160 mg, $0.60 \mathrm{mmol}$ dissolved in $50 \mathrm{ml}$ of methanol), crude ester was synthesized (133 $\mathrm{mg}$ ). After chromatography (hexane/ethyl acetate 4:1) pure compound 13a was isolated as an oil (80 mg, 50\%). ${ }^{\mathrm{l}} \mathrm{H}-\mathrm{NMR}(100 \mathrm{MHz}$, $\left.\mathrm{CDCl}_{3}\right): \delta 1.41\left(\mathrm{hrd}, 1 \mathrm{H}, \mathrm{OCH}_{2} \mathrm{C} \underline{\mathrm{H}} \mathrm{HCHO}\right), 2.04$ (dq. $1 \mathrm{H}, \mathrm{OCH}_{2} \mathrm{CH} \underline{\mathrm{CH}} \mathrm{O}$. J $13 \mathrm{~Hz}$ and $5.3 \mathrm{~Hz}$ ), 2.61 (d, $1 \mathrm{H}$, $\mathrm{OH}, \mathrm{J} 4.0 \mathrm{~Hz}$ ), $3.75\left(\mathrm{~s}, 3 \mathrm{H}, \mathrm{OCH}_{3}\right), 3.91$ (t. IH. OCHHCH $\left.2 \mathrm{CHO}_{2} \mathrm{~J} 2.4 \mathrm{~Hz}\right), 4.03$ (t. IH. OCH $\mathrm{HCH}_{2} \mathrm{CHO}_{2} \mathrm{~J} 2.8$ $\mathrm{Hz}), 1.234 .36\left(\mathrm{~m}, 1 \mathrm{H}, \mathrm{OCH}_{2} \mathrm{CH}_{2} \mathrm{C} \underline{\mathrm{HO}}\right), 4.11-4.53(\mathrm{~m} .1 \mathrm{H} . \mathrm{CHOC} \underline{\mathrm{H}}(\mathrm{OH}) \mathrm{CH}), 5.55(\mathrm{~s}, 1 \mathrm{H}, \mathrm{CHPh}), 6.18(\mathrm{dd}$, $1 \mathrm{H}, \mathrm{CH}=\mathrm{CHC}(\mathrm{O}), \mathrm{J} 15.6 \mathrm{~Hz}$ and $1.8 \mathrm{~Hz}), 6.97(\mathrm{dd} .1 \mathrm{H}, \mathrm{CH}=\mathrm{CHC}(\mathrm{O}), \mathrm{J} 15.6 \mathrm{~Hz}$ and $4.3 \mathrm{~Hz}), 7.32-7.54(\mathrm{~m}, 5 \mathrm{H}$, Ph) ppm. IR $\left(\mathrm{CCl}_{4}\right):$ v 3590, 3060, 3030, 2965. 2950, 2870.1725.1665.1435, 1310.1170, 1110.700 $\mathrm{cm}^{-1}$. MS (CI): $\left.m / e(\%) 279\left(7, \mathrm{M}^{+}+1\right), 247(3,-\mathrm{MeOH}), 173(18,-\mathrm{PhClO}) \mathrm{H}\right), 163(100), 155\left(43 .-\mathrm{PhC}(\mathrm{O}) \mathrm{H},-\mathrm{H}_{2} \mathrm{O}\right), 141$ (2), $-\mathrm{PhC}(\mathrm{O}) \mathrm{H},-\mathrm{MeOH}), 123$ (10, $\left.-\mathrm{PhC}(\mathrm{O}) \mathrm{H} . \mathrm{MeOH},-\mathrm{H}_{2} \mathrm{O}\right), 107(20), 91(24), 79(14), 57(11), 41$ (22). EI/HRMS: m/e calcd. for $\mathrm{C}_{15} \mathrm{H}_{18} \mathrm{O}_{5} 278.11542$. found $278.11544 \pm 0.00081$ a.m.u.

( $(2 R, 3 S)-3-[(2 S, 4 S)-2-p h e n v-1,3-d i o x a n-4-1 /)$ xiran-2-1/methand $(\mathbf{1 0 b})$ :

Starting with allylic alcohol $9(0.95 \mathrm{~g}, 4.3 \mathrm{mmol})$, following the procedure for the synthesis of 4 a and using D-(-)-DET as chiral inductor, crude epoxy alcohol was prepared (1.013 g), which was recrystallized from ether at

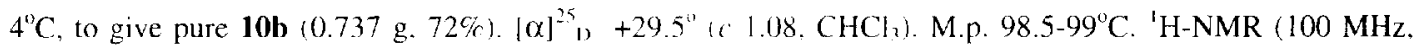
$\left.\mathrm{CDCl}_{3}\right): \delta 1.42-1.82\left(\mathrm{~m}, 2 \mathrm{H}, \mathrm{OCH}_{2} \mathrm{CH} \underline{\mathrm{HCHO}}\right.$ and $\left.\mathrm{OH}\right), 2.05$ (dq. $1 \mathrm{H}, \mathrm{OCH}_{2} \mathrm{CHHCHO}_{1} \mathrm{~J} 12 \mathrm{~Hz}$ and $\left.5.2 \mathrm{~Hz}\right)$,

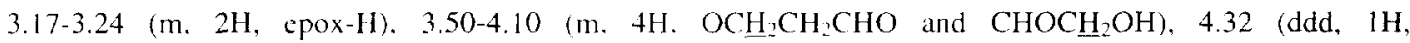
$\mathrm{OCH}_{2} \mathrm{CH}_{2} \mathrm{CHO}, \mathrm{J} 11.5 \mathrm{~Hz}, 4.6 \mathrm{~Hz}$ and $\left.1.4 \mathrm{~Hz}\right) .5 .52(\mathrm{~s}, 1 \mathrm{H}, \mathrm{CHPh}), 7.31-7.55(\mathrm{~m}, 5 \mathrm{H}, \mathrm{Ph}) \mathrm{ppm}$. IR $\left(\mathrm{CCl}_{4}\right): v$ $3600,3400,3050,2970,2930,2870.1110 \mathrm{~cm}^{\prime}$. MS (CI): m/e $\left.1 \%\right) 237\left(7, \mathrm{M}^{+}+1\right), 236\left(33, \mathrm{M}^{+}\right), 235\left(48, \mathrm{M}^{+}\right.$ -1). $\left.163\left(65,-\mathrm{CHOCHCH}_{2} \mathrm{OH}\right), 131(8 .-\mathrm{PhC}(\mathrm{O}) \mathrm{H})\right), 113\left(45,-\mathrm{PhC}(\mathrm{O}) \mathrm{H},-\mathrm{H}_{2} \mathrm{O}\right), 107\left(71, \mathrm{PhC}(\mathrm{O}) \mathrm{H}^{+}+1\right), 106$ $\left(30, \mathrm{PhC}(\mathrm{O}) \mathrm{H}^{+}\right), 105\left(100, \mathrm{PhC}(\mathrm{O})^{+}\right), 95(23), 91(42), 87(63), 83(61), 79(47), 77(46), 71(63), 67(35), 57$ (46). 41 (57). El/HRMS: m/e calcd. for $\mathrm{C}_{13} \mathrm{H}_{16} \mathrm{O}_{4} 236.1049$. found $236.10496 \pm 0.00046$ a.m.u. Calcd. for $\mathrm{C}_{13} \mathrm{H}_{16} \mathrm{O}_{4}$ : C 66.09. H 6.83\%, found $\mathrm{C} 66.53, \mathrm{H} 6.88 \%$. 
((2S,3S)-3-[(2S,4S)-2-phenyl-1,3-dioxan-4-ylloxiran-2-yl/carboxylic acid (11b):

Glycidic acid 11b was prepared following the procedure for the synthesis of compound 11a. Starting with epoxy alcohol $10 \mathrm{~b}$ ( $500 \mathrm{mg}, 2.12 \mathrm{mmol}$ ) crude aldehyde was prepared $(610 \mathrm{mg}) .{ }^{1} \mathrm{H}-\mathrm{NMR}\left(100 \mathrm{MHz}, \mathrm{CDCl}_{3}\right)$ : $\delta 1.56$ (br d, $1 \mathrm{H}, \mathrm{OCH}_{2} \mathrm{CHHCHO}, \mathrm{J} 13 \mathrm{~Hz}$ ), 2.05 (dq, $1 \mathrm{H}, \mathrm{OCH}_{2} \mathrm{CH} \underline{\mathrm{HCHO}}, \mathrm{J} 11.7$ and $5.2 \mathrm{~Hz}$ ), $3.41-3.48$ (m, 2H. epox-H), 3.83-4.09 (m. 2H. OCH ${ }_{2} \mathrm{CH}_{2} \mathrm{CHO}$ ), 4.33 (ddd, $1 \mathrm{H}, \mathrm{OCH}_{2} \mathrm{CH}_{2} \mathrm{CHO}, \mathrm{J} 11.6 \mathrm{~Hz}, 5.2 \mathrm{~Hz}$ and 1.4 $\mathrm{Hz}$ ), $5.5 \mathrm{l}(\mathrm{s}, 1 \mathrm{H} . \mathrm{CHPh}), 7.31-7.54(\mathrm{~m}, 5 \mathrm{H}, \mathrm{Ph}), 9.06(\mathrm{~d}, 1 \mathrm{H}, \mathrm{C}(\mathrm{O}) \mathrm{H}, \mathrm{J} 6 \mathrm{~Hz}) \mathrm{ppm}$. IR $\left(\mathrm{CCl}_{4}\right): v 3090,3070$, $3030,2970,2920,2850,1735,700,650 \mathrm{~cm}^{-1}$. The aldehyde $(600 \mathrm{mg})$ was oxidized in a mixture of tert-butyl alcohol ( $50 \mathrm{ml}$ ), 2-methyl-2-butene $(12 \mathrm{ml}$ ) and dichloromethane ( $2 \mathrm{ml}$, cf. compound $\mathbf{1 1 a}$ ), yielding acid $\mathbf{1 1 b}$ as a yellow oil (250 mg), which was innmediately converted into the epoxy diazo ketone. 'H-NMR (90 MHz, $\mathrm{CDCl}_{3}$ ): $\delta 1.45$ (br d, $1 \mathrm{H}, \mathrm{OCH}_{2} \mathrm{C} \underline{\mathrm{H}} \mathrm{HCHO}, \mathrm{J} 12 \mathrm{~Hz}$ ), 1.63-2.17 (m, 1H, OCH $2 \mathrm{CH} \underline{\mathrm{HCHO}}$ ), 3.30-3.60 (m, 2H, cpox-H), 3.65-4.05 (m, 2H. OC $\underline{\mathrm{H}}_{2} \mathrm{CH}_{2} \mathrm{CHO}$ ), 4.05-4.33 (m. 1H, OCH $\mathrm{CH}_{2} \mathrm{CHO}$ ), 5.46 (s, 1H, CHPh), 7.30$7.55(\mathrm{~m}, 5 \mathrm{H}, \mathrm{Ph}), 8.77\left(\mathrm{br} \mathrm{s}, 1 \mathrm{H}, \mathrm{CO}_{2} \mathrm{H}\right) \mathrm{ppm}$. IR $\left(\mathrm{CCl}_{4}\right): v 3600-2700,2950,2920,2860,1725 \mathrm{~cm}^{-1}$.

$1-\{(2 S, 3 S)-3-/(2 S, 4 S)-2$-phenyl-1,3-dioxan-4-ylloxiran-2-yl\}-2-diazo-ethanone $(\mathbf{1 2 b})$ :

Following the procedure for the synthesis of compound 5a, crude diazo ketone 12b was prepared (137 $\mathrm{mg}$ ), starting from crude glycidic acid $11 \mathbf{b}(180 \mathrm{mg})$. Chromatography of the crude product (hexane/ethyl acetate $1: 1)$ yielded diazo compound $\mathbf{1 2 b}$ as an oil $(58 \mathrm{mg}, 30 \%)$. The low yield was due to decomposition on the silica gel column (isolation of benzaldehyde). ${ }^{1} \mathrm{H}-\mathrm{NMR}\left(100 \mathrm{MHz}, \mathrm{CDCl}_{3}\right.$ ): $\delta 1.47-1.65$ (br d, $1 \mathrm{H}, \mathrm{OCH}_{2} \mathrm{CHHCHO}, \mathrm{J} 13$ $\mathrm{Hz}), 2.04\left(\mathrm{dq}, 1 \mathrm{H}, \mathrm{OCH}_{2} \mathrm{CH} \underline{\mathrm{HCIO}}, \mathrm{J} 11.5 \mathrm{Iz}\right.$ and $5.2 \mathrm{Iz}$ ), 3.20 (dd, $1 \mathrm{H}, \mathrm{CHOCHCH}(\mathrm{O}), \mathrm{J} 5 \mathrm{~Hz}$ and $2 \mathrm{~Hz}$ ), 3.51 (d, $1 \mathrm{H}, \mathrm{CHOCHCH}(\mathrm{O}), \mathrm{J} 2 \mathrm{~Hz}), 3.76-3.90\left(\mathrm{~m}, 1 \mathrm{H}, \mathrm{OCHHCH}_{2} \mathrm{CHO}\right), 4.01$ (dd, 1H, OCH $11.8 \mathrm{~Hz}$ and $2.7 \mathrm{~Hz}$ ), 4.31 (ddd, $1 \mathrm{H}, \mathrm{OCH}_{2} \mathrm{CH}_{2} \mathrm{CHO}$. J $11.4 \mathrm{~Hz}, 5.1 \mathrm{~Hz}$ and $1.3 \mathrm{~Hz}$ ), 5.50 (s, 2H, CHPh and $\left.\mathrm{CHN}_{2}\right), 7.31-7.53(\mathrm{~m}, 5 \mathrm{H} . \mathrm{Ph}) \mathrm{ppm}$. IR $\left(\mathrm{CCl}_{4}\right): \vee 3120,3070,3040,2960,2930,2860,2110,1645,1370 \mathrm{~cm}^{-1}$. MS (CI): m/e (\%) $275\left(14, \mathrm{M}^{+}+1\right), 273\left(\mathrm{M}^{+}-1\right), 197(6), 163(18), 131(7), 124(12), 107\left(20, \mathrm{PhC}(\mathrm{O}) \mathrm{H}^{+}+1\right), 106$ (14. $\left.\mathrm{PhC}(\mathrm{O}) \mathrm{H}^{+}\right) .105\left(100, \mathrm{PhC}(\mathrm{O})^{+}\right), 91$ (19). 83 (25), 77 (19), 69 (23), 55 (26), 43 (20), 41 (40). EL/HRMS: m/e calcd. for $\mathrm{C}_{14} \mathrm{H}_{14} \mathrm{~N}_{2} \mathrm{O}_{4} 274.09536$, found $274.09550 \pm 0.00081$ a.m.u.

Ethyl E-4-f(2S,4S)-2-phenyl-J,3-dioxan-4-y//-4-(S)-hydroxy-but-2-enoate (13b):

Starting with diazo ketone $12 \mathrm{~b}$ ( $58 \mathrm{mg}, 0.21 \mathrm{mmol}$ ) and following the procedure for the synthesis of compound 7a, unsaturated ester 13b was obtained as an oil (52 mg. 75\%) in absolute methanol as the solvent. 'H-NMR $\left(100 \mathrm{MHz}, \mathrm{CDCl}_{3}\right.$ ): $\delta 1.52$ (br d. $1 \mathrm{H} . \mathrm{OCH}_{2} \mathrm{CH}$ HCHO. J $13 \mathrm{~Hz}$ ). 1.98 (dq, $1 \mathrm{H}, \mathrm{OCH}_{2} \mathrm{CHHCHO}_{1} \mathrm{~J} 11.6 \mathrm{~Hz}$ and $5.2 \mathrm{~Hz}$ ), 2.82 (d, III. OII. J $4.1 \mathrm{~Hz}$ ), 3.75 (s, 3H, $\left.\mathrm{OCH}_{3}\right), 3.76-3.86\left(\mathrm{~m}, 1 \mathrm{H}, \mathrm{OCHHCH}_{2} \mathrm{CHO}\right), 3.99$ (dd, 1H, $\mathrm{OC}_{\mathrm{H}} \mathrm{HCH}_{2} \mathrm{CHO}, \mathrm{J} 11.9 \mathrm{~Hz}$ and $\left.2.8 \mathrm{~Hz}\right), 4.18-4.38\left(\mathrm{~m}, 2 \mathrm{H} . \mathrm{OCH}_{2} \mathrm{CH}_{2} \mathrm{CHO}\right.$ and $\left.\mathrm{CHOCH}(\mathrm{OH})\right), 5.53(\mathrm{~s}, 1 \mathrm{H}$, $\mathrm{CHPh}), 6.21$ (dd, $1 \mathrm{H}, \mathrm{CH}=\mathrm{CHC}(\mathrm{O}), \mathrm{J} 15.6 \mathrm{~Hz}$ and $1.7 \mathrm{~Hz}), 6.95(\mathrm{dd}, \mathrm{IH}, \mathrm{C} \underline{\mathrm{H}}=\mathrm{CHC}(\mathrm{O}), \mathrm{J} 15.6 \mathrm{~Hz}$ and $4.8 \mathrm{~Hz})$, $7.32-7.54(\mathrm{~m}, 5 \mathrm{H}, \mathrm{Ph}) \mathrm{ppm}$. IR $\left(\mathrm{CCl}_{4}\right): v 3620,3400,2970,2920,2880,1725 \mathrm{~cm}^{-1}$. MS (CI): m/e (\%) $279(2$, $\left.\mathrm{M}^{+}+1\right), 247(2,-\mathrm{MeOH}), 223(2), 163\left(47,-\mathrm{CH}(\mathrm{OH}) \mathrm{CH}=\mathrm{CHCO}{ }_{2} \mathrm{Me}\right), \mathrm{I} 55\left(31,-\mathrm{PhC}(\mathrm{O}) \mathrm{H},-\mathrm{H}_{2} \mathrm{O}\right), 149(32), 141$ (53. $-\mathrm{PhC}(\mathrm{O}) \mathrm{H},-\mathrm{MeOH}), 123\left(18,-\mathrm{PhC}(\mathrm{O}) \mathrm{H},-\mathrm{MeOH},-\mathrm{H}_{2} \mathrm{O}\right), 116(25), 105\left(36, \mathrm{PhC}(\mathrm{O})^{+}\right), 86(51), 75(16), 57$ (36). 41 (100). EL/HRMS: m/e calcd. for $\mathrm{C}_{15} \mathrm{H}_{18} \mathrm{O}_{5} 278.11542$, found $278.11517 \pm 0.00081$ a.m.u. 


\section{REFERENCES}

1. Van Haard, P.M.M.; Thijs, L.; Zwanenburg, B., Tetrahedron Lett., 1975, 803

2. Thijs, L.; Dommerholt, F.J.; Leemhuis, F.M.C.; Zwanenburg, B., Tetrahedron Lett., 1990, 31, 6589

3. a. Katsuki. T.; Sharpless, K.B., J. Am. Chem. Soc., 1980, 102, 5974: b. Gao. Y.; Hanson, R.M.; Klunder, J.M.; Ko, S.Y.; Masamune, H.; Sharpless, K.B., J. Am. Chem. Sac., 1987, 109, 5765

4. Thijs, L.; Waanders, P.P.; Stokkingreef, E.H.M.; Zwanenburg, B., Rec. Trav. Chim. Pays-Bas, 1986, 105, 332

5. Waanders, P.P.; Thijs, L.; Zwanenburg, B., Tetrahedron Lett., 1987, 28, 2409

6. Dommerholt, F.J.; Thijs, L.; Zwanenburg, B., Tetrahedron Lett., 1991, 32, 1499

7. Dommerholt, F.J.; Thijs, L.; Zwanenburg, B., Tetruhedron Lett., 1991, 32, 1495

8. Thijs, L.; Egenberger, D.M.; Zwanenburg, B., Tetrahedron Lett., 1989, 30,2153

9. Leemhuis, F.M.C.; Thijs, L.; Zwanenburg, B., J. Org. Chem., 1993, 58, 7170

10. Thijs, L.; Stokkingreef, E.H.M.; Lemmens, J.M.; Zwanenburg, B., Tetrahedron, 1985, 30, 2949

11. Saito, T.; Thijs, L.; Ettema, G.-J.; Zwanenburg, B., Tetrahedron Lett., 1993, 34, 3589

12. Kierstead, R.W.; Faraone, A.; Mennona, F.; Mullin, J.; Guthrie, R.W.; Crowley, H.; Simko, B.; Blaber, L.C., J. Med. Chem., 1983, 26, 1561

13. Jackson, D.Y., Synth. Comm., 1988, 18, 337

14. Blanchette, M.A.; Choy, W.; Davis, J.T.: Essenfeld, A.P.; Masamune, S.; Roush, W.R.; Sakai, T., Tetrahedron Lett., 1984, 25, 2183

15. Minami, N.; Ko, S.; Kishi, Y., J. Am. Chem. Soc. 1982, 104. 1109

16. Katsuki, T.; Lee, A.W.M.; Ma. P.; Martin, V.S.; Masamune, S.; Sharpless, K.B.; Tuddenham, D.; Walker, F.J., J. Org. Chem., 1982, 47, 1373

17. Scheuplein, S.W.; Kusche, A.: Brückner. R.: Harms, K., Chem. Ber., 1990, 123, 917

18. Ko, S.Y.; Lee, A.W.M.: Masamune, S.; Reed, L.A.: Sharpless, K.B.; Walker, F.J., Tetrahedron, 1990, 46, 245

19. Carlsen, P.M.J.; Katsuki, T.; Martin, V.S.: Sharpless, K.B., J. Org. Chem., 1981, 46, 3936

20. Ratcliffe, R.; Rodehurst, R., J. Org. Chem., 1970. 35. 4000

21. Corey, E.J.; Schmidt, G., Tetrahedron Lett., 1979. 399

22. Regeling, H.; Chittenden, G.J.F.. Rec. Trav. Chim. Pavs-Bas, 1989. 108, 330

23. Jurczak, J.; Pikul, S.; Bauer, T., Tetrahedron, 1986, 42, 447

24. Hanessian, S.; Ugolini, A.; Dubé, D.; Glamyan, A., Can.J. Chem., 1984, 62, 2146

25. a. Corcoran, R.C., Tetrahedron Lett., 1990, 31, 2101; b. Pawlak, J.; Nakanishi, N.; Iwashita, T.: Borowski, E., J. Org. Chem., 1987, 52, 289; c. Thian, M.; Slassi, A.; Chastrette, F.; Amouroux, R., Synth. Comm., 1992, 22, 83

26. a. Labelle, M.; Guindon, Y., J. Am. Chem. Soc., 1989, II1, 2204; b. Mancuso, A.J.; Huang, S.-L.; Swern, D., J. Org. Chem., 1978, 43, 2480

27. Bal, B.S.; Childers Jr., W.E.: Pinnick, H.W., Tetrahedron, 1981, 37, 2091 
Acknowledgment. $400 \mathrm{MHz}$ NMR-spectroscopy, mass spectrometry and elemental analysis were performed by Mr. A. Swolfs, Mr. P.M. Van Galen and Mrs. H. Amatdjais. This investigation was supported by the Netherlands Foundation for Chemical Research ( $\mathrm{SON}$ ) with financial aid from the Netherlands Organization for Scientific Research (NWO).

(Received in UK 25 May 1995: revised 6 July 1995: accepted 7 July 1995) 